Liposomes containing glycocholate as potential oral insulin delivery systems: preparation, in vitro characterization, and improved protection against enzymatic degradation

\author{
This article was published in the following Dove Press journal: \\ International Journal of Nanomedicine \\ 7 June 2011 \\ Number of times this article has been viewed
}

\section{Mengmeng $\mathrm{Niu}^{\prime}$ \\ Yi Lu' \\ Lars Hovgaard ${ }^{2}$ \\ Wei Wu'}

'School of Pharmacy, Fudan University, Shanghai, People's Republic of China; ${ }^{2}$ Oral Formulation Development, Novo Nordisk A/S, Maalov, Denmark
Correspondence: Wei Wu Department of Pharmaceutics, School of Pharmacy, Fudan University, 826 Zhangheng Road, Shanghai 201203, People's

Republic of China

$\mathrm{Tel}+862151980002$

Fax +862151980002

Email wuwei@shmu.edu.cn
Background: Oral delivery of insulin is challenging and must overcome the barriers of gastric and enzymatic degradation as well as low permeation across the intestinal epithelium. The present study aimed to develop a liposomal delivery system containing glycocholate as an enzyme inhibitor and permeation enhancer for oral insulin delivery.

Methods: Liposomes containing sodium glycocholate were prepared by a reversed-phase evaporation method followed by homogenization. The particle size and entrapment efficiency of recombinant human insulin (rhINS)-loaded sodium glycocholate liposomes can be easily adjusted by tuning the homogenization parameters, phospholipid:sodium glycocholate ratio, insulin:phospholipid ratio, water:ether volume ratio, interior water phase $\mathrm{pH}$, and the hydration buffer $\mathrm{pH}$.

Results: The optimal formulation showed an insulin entrapment efficiency of $30 \% \pm 2 \%$ and a particle size of $154 \pm 18 \mathrm{~nm}$. A conformational study by circular dichroism spectroscopy and a bioactivity study confirmed the preserved integrity of rhINS against preparative stress. Transmission electron micrographs revealed a nearly spherical and deformed structure with discernable lamella for sodium glycocholate liposomes. Sodium glycocholate liposomes showed better protection of insulin against enzymatic degradation by pepsin, trypsin, and $\alpha$-chymotrypsin than liposomes containing the bile salt counterparts of sodium taurocholate and sodium deoxycholate.

Conclusion: Sodium glycocholate liposomes showed promising in vitro characteristics and have the potential to be able to deliver insulin orally.

Keywords: liposomes, glycocholate, insulin, enzymatic degradation, oral

\section{Introduction}

Nonparenteral delivery is by far the most convenient route for drug delivery, especially when repeated or routine administration is necessary. ${ }^{1}$ However, the oral route, as a whole, presents a major barrier to macromolecular drugs, the oral bioavailability of which is invariably well below the therapeutic level. ${ }^{2}$ This limitation is especially pronounced for proteins and peptides because of their gastric instability, susceptibility to enzymatic degradation in the gastrointestinal tract, and low permeability across the intestinal epithelium. ${ }^{3,4}$ In order to improve the oral bioavailability of peptides, researchers have employed a variety of strategies, including enhancing permeation by absorption enhancers ${ }^{5}$ and cell-penetrating peptides, ${ }^{6}$ or by opening the tight junction of the epithelia, ${ }^{7}$ protection from gastric and enzymatic destabilization, ${ }^{8,9}$ and 
enhancing lymphatic absorption through uptake by $\mathrm{M}$ cells in the Peyer's patches. ${ }^{10}$ Multiple mechanisms are usually employed to enhance the oral bioavailability of these drugs, for example, in the design of particulate or colon-specific delivery systems for protein and peptide drugs. ${ }^{11-16}$

Liposomes, being among the most studied particulate carrier systems, have shown appealing potential in enhancing oral bioavailability of proteins and peptides. ${ }^{17-19}$ However, the efficacy of conventional liposomes, mainly composed of phospholipid and cholesterol, has been compromised due to the instability of liposomes in the gastrointestinal tract and poor permeability across the epithelial membrane. ${ }^{20}$ Modification of the composition and properties of liposomes is effective in improving the stability and uptake of protein and peptide drugs by the gastrointestinal tract. ${ }^{21}$ Recently, liposomes containing bile salts such as sodium deoxycholate, also called "bilosomes," have shown promising potential in oral immunization. ${ }^{22}$ Oral administration of bilosomes incorporating vaccines such as the influenza subunit vaccine and cholera toxin B subunit was found to create as potent an antibody response as an equivalent dose administered parenterally. ${ }^{23,24}$ Hepatitis B surface antigen-loaded bilosomes produced both a systemic as well as a mucosal antibody response upon oral administration. ${ }^{25}$ It is reported that although bile salts act exteriorly to destabilize liposomal membranes, bile salts embedded in the lipid bilayers act to stabilize lipid vesicles against further destabilization by physiological bile salts in the gastrointestinal tract. ${ }^{26,27}$ On the other hand, bile salts presenting in the lipid bilayers of the bilosomes are assumed to increase the fluidity of epithelial membranes and contribute to enhanced permeation of the vesicles. ${ }^{28}$ These ideas sparked our interest in the oral delivery of protein and peptide drugs using liposomes containing bile salts.

In this study, we investigated the feasibility of using liposomes containing bile salts to enhance the oral bioavailability of insulin, a model protein drug. More specifically, sodium glycocholate was used as a new bile salt in the liposomal formulation to strengthen the enzyme-inhibiting and permeation-enhancing effect. It is reported that sodium glycocholate has good permeation-enhancing properties, ${ }^{29}$ with relatively low toxicity, ${ }^{30}$ as well as a protease-inhibiting effect. ${ }^{31}$ We hypothesized that liposomes containing sodium glycocholate would provide increased protection of insulin from enzymatic degradation and enhanced permeation across the gastrointestinal epithelium. In this first report, liposomes containing sodium glycocholate were prepared by a reversed-phase evaporation method and characterized in vitro.

\section{Methods and materials Materials}

Recombinant human insulin (rhINS, 27 units/mg) was provided by Novo Nordisk (Copenhagen, Denmark). Sodium glycocholate was purchased from Amresco (Solon, OH). Soybean phosphotidylcholine and cholesterol was supplied by Lipoid (Ludwigshafen, Germany). Sodium taurocholate and sodium deoxycholate were obtained from Sigma (Shanghai, China). Pepsin (Ph Eur) and trypsin (more than 6000 BAEE units/mg) were purchased from Sigma (Shanghai, China). $\alpha$-chymotrypsin (more than 1500 USP units $/ \mathrm{mg}$ ) were obtained from BD Falcon (New Jersey, USA). A Sephadex G50 column was purchased from Pharmacia (Shanghai, China). Deionized water was prepared by a Milli-Q purification system (Molsheim, France). High-pressure liquid chromatography (HPLC) grade acetonitrile was supplied by Merck (Darmstadt, Germany). All other chemicals were of analytical grade and used as received.

Four-week-old male Kunming mice weighing 18-20 g were purchased from the Animal Center at the Shanghai University of Traditional Chinese Medicine (Shanghai, China) and were raised in rooms controlled at $23 \pm 1{ }^{\circ} \mathrm{C}$ and $55 \% \pm 5 \%$ relative humidity with 12 -hour light $/ 12$-hour dark time cycles. The mice received a standard laboratory chow diet and tap water during acclimatization. All experimental protocols concerning the handling of mice were in accordance with the requirements of the Institutional Animal Care and Use Committee at Fudan University.

\section{Preparation of insulin-loaded liposomes}

rhINS-loaded liposomes containing sodium glycocholate were prepared by a reversed-phase evaporation method. Briefly, soybean phosphotidylcholine and sodium glycocholate were dissolved in absolute ether, into which rhINS citric- $\mathrm{Na}_{2} \mathrm{HPO}_{4}$ buffer solution ( $\mathrm{pH} 3.0$, if not specified otherwise) was added drop by drop. The mixture was sonicated for 5 minutes in a water bath until a w/o emulsion was formed. The emulsion was rota-evaporated under $0.07-0.08 \mathrm{mPa}$ pressure with a rotating speed of $50 \mathrm{rpm}$ at $30^{\circ} \mathrm{C}$ for 20 minutes to remove the organic solvent. A citric acid- $\mathrm{Na}_{2} \mathrm{HPO}_{4}$ buffer ( $\mathrm{pH}$ 5.6) was then added to hydrate the dry lipids until a homogeneous dispersion was formed. Finally, this dispersion was extruded through a high-pressure homogenizer (AH 100 D; ATS Engineering Inc, Brampton, ON) to obtain the sodium glycocholate liposomes. Before assaying, free rhINS was removed by eluting the liposome dispersion through the Sephadex G50 column, following similar procedures as described below for measurement of entrapment efficiency. 
To prepare rhINS-loaded liposomes containing sodium taurocholate or sodium deoxycholate, similar procedures were followed, but substituting sodium glycocholate for sodium taurocholate or sodium deoxycholate, and likewise for conventional liposomes composed of soybean phosphotidylcholine and cholesterol as a control. Blank liposomes were prepared following the same procedures without adding rhINS.

\section{Particle size analysis}

Sizes of the sodium glycocholate liposomes were examined using a dynamic light scattering instrument (NICOMP 380 ZLS Particle Sizing Systems; PSS, Inc, Santa Barbara, CA). Liposomes were placed in a disposable cuvette, and photon counts were collected over 5 minutes using an angle of $90^{\circ}$ at $25^{\circ} \mathrm{C}$. Particle size was analyzed by the ZPW388 software program (PSS, Inc) and expressed with intensity-based Gaussian distribution. The result was expressed as mean \pm standard deviation $(n=3)$.

\section{Entrapment efficiency}

Entrapment efficiency of the rhINS in sodium glycocholate liposomes was determined after separating free rhINS from the liposomes using molecular exclusion chromatography with a $30 \mathrm{~cm}$ long Sephadex G50 column. Briefly, liposome samples were introduced into the column and subsequently eluted with citric acid- $\mathrm{Na}_{2} \mathrm{HPO}_{4}$ buffer ( $\mathrm{pH}$ 5.6). The eluted fraction containing the entrapped liposomal rhINS was analyzed by HPLC as described below. The entrapment efficiency was defined as the ratio of liposome-associated rhINS ( rhINS $_{\text {liposomal }}$ ) to total rhINS ( rhINS $_{\text {total }}$ ), and calculated as shown in Eq (1):

$$
E E(\%)=\frac{r h I N S_{\text {liposomal }}}{r h I N S_{\text {total }}} \times 100 \%
$$

\section{Determination of insulin}

rhINS was assayed by a reversed-phase HPLC/ultraviolet method. ${ }^{32}$ The Agilent 1100 series HPLC system (Agilent Technologies, Inc, Santa Clara, CA) was composed of a quaternary pump, a degasser, an autosampler, a column heater, and a tunable ultraviolet detector. rhINS was separated at $25^{\circ} \mathrm{C}$ using a $\mathrm{C} 18$ column (Zorbax ${ }^{\circledR}$, $5 \mu \mathrm{m}, 4.6 \mathrm{~mm} \times 150 \mathrm{~mm}$, Agilent) with a C18 precolumn $(2 \mathrm{~mm} \times 20 \mathrm{~mm})$, and detected by measuring the $220 \mathrm{~nm}$ absorbance of the eluate. The mobile phase was a mixture of acetonitrile and $0.57 \% \mathrm{H}_{3} \mathrm{PO}_{4}$ (adjusted to $\mathrm{pH} 2.25$ with triethylamine) in a volume ratio of $26 / 74$, pumped at a flow rate of $1.0 \mathrm{~mL} / \mathrm{min}$.

\section{Conformational stability}

The conformational stability of rhINS was studied by circular dichroism spectroscopy. To prepare samples for the circular dichroism test, rhINS was released by disrupting the liposomes using 1\% Triton X-100. rhINS was then separated from soybean phosphotidylcholine and sodium glycocholate using an ENVI-18 solid-phase extraction column (Supelco, Bellefonte, PA) to avoid interference. The separation details were as follows. A $1 \mathrm{~mL}$ sample of destabilized liposomal mixture was applied onto an ENVI-18 column which had been activated by methanol. Elution was then performed with $1 \mathrm{~mL}$ aqueous methanol (40\%) to wash out sodium glycocholate and soybean phosphotidylcholine. After that, 60\% aqueous methanol was added to elute the rhINS, which was collected and adjusted to a concentration of approximately $20 \mu \mathrm{g} / \mathrm{mL}$ for the circular dichroism test. A rhINS solution of equal concentration, dissolved in $60 \%$ aqueous methanol, was used as the control. The conformational characteristics of the insulin released and the control were examined by circular dichroism on a Jasco-715 circular dichroism spectrophotometer (Jasco Co Ltd, Hachioji, Japan). Five scans were performed and averaged for each sample.

\section{Bioactivity}

rhINS solution in $0.9 \% \mathrm{NaCl}$ solution was used as a positive control and $0.9 \% \mathrm{NaCl}$ solution as a negative control. The control and rhINS solutions retrieved from the different formulations as described earlier were injected subcutaneously into normal mice at a dose of $0.5 \mathrm{IU} / \mathrm{kg}$. Blood samples were collected from the retro-orbital plexus before dosing and 1 hour after dosing, and then centrifuged (without addition of anticoagulants) at $4000 \mathrm{~g}$ for 5 minutes. Serum was collected and blood glucose levels were determined using a glucose GOD-PAD kit (Shanghai Rongsheng Biotech Co Ltd, Shanghai, China). Bioactivity was expressed as the reduction ratio of postdosing to predosing blood glucose level.

\section{Transmission electron microscopy}

The morphology of the liposomes was studied by negative transmission electron microscopy following a standard procedure. ${ }^{33}$ Briefly, a drop of liposome dispersion was placed on 300-mesh and carbon-coated copper grids, and allowed to adsorb. The surplus was removed using blotting paper. A drop of $1 \%$ phosphotungstic acid was added, and the liposomes were stained for 30-60 seconds. The stained 
liposomes were allowed to dry in ambient conditions and inspected with transmission electron microscopy (JEM-1230; JEOL, Tokyo, Japan) at an acceleration voltage of $120 \mathrm{kV}$. The micrographs were recorded at a final magnification of $60,000 \times$

\section{Leakage of insulin}

The leakage of rhINS from the sodium glycocholate liposomes was measured by detecting the change in entrapment efficiency change at different time intervals. Briefly, rhINSloaded liposomes were suspended in pH 2.0, 5.6, and 6.8 citric acid- $\mathrm{Na}_{2} \mathrm{HPO}_{4}$ buffers. The suspensions were placed in a $37^{\circ} \mathrm{C}$ water bath and shaken at $60 \mathrm{rpm}$ in a reciprocal shaker (SHZ-C, Pudong Physical Optical Co Ltd, Shanghai, China) over 6 hours. At specific time intervals, the entrapment efficiency of the liposomes was detected using the analytical method described earlier. Sink conditions were maintained through the study.

\section{Protection of insulin from enzymatic digestion}

The protective effect on rhINS-loaded sodium glycocholate liposomes was studied using a dissolution tester (ZRS-8G; Tianda Technology Co Ltd, Tianjin, China) following procedures similar to those described in the Chinese Pharmacopoeia (2010) for dissolution testing by the small beaker method. Briefly, $0.5 \mathrm{~mL}$ of liposome suspension was diluted in $50 \mathrm{~mL}$ of digestive media and subjected to enzymatic degradation. The digestive media comprised either simulated gastric fluid (containing $1 \%$ pepsin, $\mathrm{pH} 1.2$ ) or simulated intestinal medium (containing $1 \%$ trypsin, $\mathrm{pH} 6.8$ ), or $\alpha$-chymotrypsin solution $(100 \mu \mathrm{g} / \mathrm{mL}$, in phosphate buffer, $\mathrm{pH}$ 7.8). The temperature was maintained at $37 \pm 1.0^{\circ} \mathrm{C}$ and stirred with a paddle at $100 \mathrm{rpm}$. At appropriate time intervals, $200 \mu \mathrm{L}$ of the suspension was withdrawn and diluted with an equal volume of $0.1 \mathrm{M} \mathrm{NaOH}$ for simulated gastric fluid, $0.1 \mathrm{M}$ $\mathrm{HCl}$ for simulated intestinal medium, and $\alpha$-chymotrypsin solution to terminate degradation. Samples were subsequently treated with Triton $\mathrm{X}-100$ to release rhINS from the liposomes, prior to HPLC assay.

\section{Statistical analysis}

The results were expressed as means \pm standard deviations. One-way analysis of variance was performed to assess the significance of the differences between the data. Results with $P<0.05$ were considered to be statistically significant.

\section{Results}

\section{Preparation and characterization of rhINS sodium glycocholate liposomes}

Sodium glycocholate liposomes were obtained easily following the preparative procedures similar to our previous report using sodium deoxycholate. ${ }^{34}$ Replacing sodium deoxycholate with sodium glycocholate did not lead to significant variation in the preparative process. The primary objective of the formulation study was to optimize the experimental conditions to achieve high rhINS loading and smaller particle size, as well as robust rhINS stability during preparative stress.

Figure 1 shows the effect of the homogenization parameters, including homogenizing pressure and number of cycles, on entrapment efficiency and particle size. Increasing the homogenization pressure from 100 to 500 bar led to a significant decrease in particle size (from $430 \mathrm{~nm}$ to $100 \mathrm{~nm}$ ), and a slight decrease in entrapment efficiency from $18 \%$ to
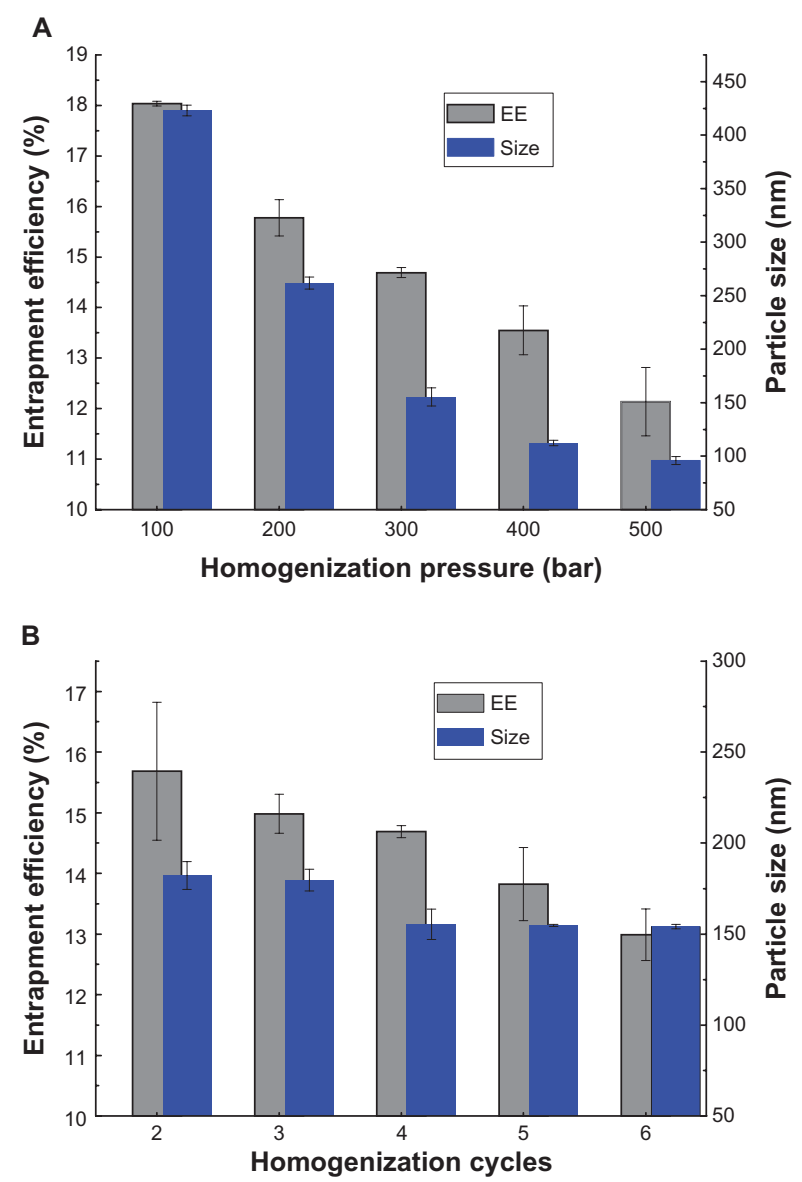

Figure I Effects of homogenization pressure (A) and number of cycles (B) on recombinant human insulin entrapment efficiency and particle size of sodium glycocholate liposomes, when cycles fixed at four and pressure 300 bar for each. Note: Data expressed as means \pm standard deviations $(n=3)$.

Abbreviation: EE, entrapment efficiency. 
$12 \%$ (Figure 1A). However, the efficiency of particle size reduction decreased when the homogenization pressure increased over 300 bar, thus 300 bar was used for the next investigation. There was no significant effect of number of homogenization cycles (from 2-6) on entrapment efficiency or particle size, although a slight decreasing trend can be observed (Figure 1B).

Figure 2 shows the effect of the formulation variables on entrapment efficiency, including soybean phosphotidylcholine:sodium glycocholate ratio, rhINS:soybean phosphotidylcholine ratio, water:ether volume ratio, $\mathrm{pH}$ value of the inner water phase, ionic strength of the hydration solution, and hydration time, when the homogenization parameters were maintained at 300 bar and four cycles. The soybean phosphotidylcholine:sodium glycocholate ratio had the most significant effect on entrapment efficiency, with a near linear increase from $3 \%$ to $25 \%$ when the soybean phosphotidylcholine:sodium glycocholate ratio increased from 1:1 to $8: 1$ (Figure 2A). It is obvious that when the total amount of soybean phosphotidylcholine and sodium glycocholate was fixed, the presence of more sodium glycocholate in the formulation compromises the entrapment of rhINS. The rhINS:soybean phosphotidylcholine ratio also seemed to have a significant effect on entrapment efficiency (Figure 2B). The entrapment efficiency was $11 \%$ at a rhINS:soybean phosphotidylcholine ratio of $0.02: 1$, which doubled when the rhINS:soybean phosphotidylcholine ratio decreased to 0.00125:1. During the preparation of the coarse w/o emulsion, rhINS is dissolved in citric acid- $\mathrm{Na}_{2} \mathrm{HPO}_{4}$ buffer. The $\mathrm{pH}$ of this buffer should be adjusted to ensure sufficient solubility and entrapment efficiency of rhINS. Comparison of entrapment efficiency obtained at different $\mathrm{pH}$ values indicated that entrapment efficiency followed a bell-shaped curve, with a maximum at pH 7.6 (Figure 2C). However, there was no significant difference observed between $\mathrm{pH} 3.0$ and $\mathrm{pH} 7.6$. Therefore, a $\mathrm{pH}$ of 3.0 was employed due to the relatively high solubility of rhINS at this $\mathrm{pH}$. The effects of the ionic strength of the hydration solution on entrapment efficiency are shown in Figure 2D. The maximum entrapment efficiency was achieved at an ionic strength of 0.6. The volume ratio of water:ether had a slight influence on entrapment efficiency, with a highest value of about $25 \%$ at $1: 5$. However, at the highest water:ether ratio of $1: 3$, the entrapment efficiency value was the smallest (below 20\%, Figure 2E). The hydration time also influenced entrapment efficiency. As can be observed in Figure 2F, entrapment efficiency increased from $11 \%$ to $15 \%(P<0.01)$ as the hydration time increased from 15 minutes to 30 minutes. A hydration time of 30 minutes was enough to achieve high entrapment efficiency because a further increase in hydration time did not lead to a further increase in entrapment efficiency.

Based on the results of the formulation study, the optimal preparative conditions were chosen as follows: $334 \mathrm{mg}$ soybean phosphotidylcholine and $66 \mathrm{mg}$ sodium glycocholate $(4: 1, \mathrm{~mol} / \mathrm{mol})$ were dissolved in $10 \mathrm{~mL}$ absolute ether, into which $2 \mathrm{~mL}$ rhINS buffered solution $(4 \mathrm{mg} / \mathrm{mL})$ was added. The mixture was sonicated and hydrated for 30 minutes to obtain a sodium glycocholate liposome dispersion. Finally, this suspension was homogenized at 300 bar for four cycles. The resulting rhINS-loaded sodium glycocholate liposomes showed an entrapment efficiency of $30.2 \pm 2.2 \%$, drug loading of $120.8 \pm 2.7 \mu \mathrm{g} / \mathrm{mL}$, and a particle size of $154 \pm 18 \mathrm{~nm}$ with a polydispersity index of $0.340 \pm 0.024$ (Figure 3).

\section{Conformational and bioactive stability}

Far-ultraviolet circular dichroism spectroscopy is usually used as a fingerprint for the identification of various secondary structural elements of peptides. ${ }^{35}$ Therefore, it was employed as an easy tool to study the conformational stability of the rhINS. Preparative variables, such as homogenization pressure and number of cycles, hydration time, amount of soybean phosphotidylcholine and insulin, and volume of organic solvents volume, at their extreme levels, may have an impact on the conformational stability of rhINS. Figure 4 shows the circular dichroism spectra of rhINS in sodium glycocholate liposomes prepared under extreme conditions, including the maximum amount of sodium glycocholate (soybean phosphotidylcholine:sodium glycocholate ratio of 2:1), maximum amount of rhINS (rhINS:soybean phosphotidylcholine ratio of 0.0025:1), maximum ether volume (water:ether ratio of 1:7), the strongest ionic strength (1.2), and the extreme preparation conditions (hydration for 90 minutes; homogenization at 500 bar for six cycles), as well as a combination of the extreme conditions. The circular dichroism spectrum of rhINS released from the optimal sodium glycocholate liposome formulation was also recorded and compared with rhINS solution (Figure 4, insert).

The circular dichroism spectra for free rhINS in solution show a valley at $208 \mathrm{~nm}$ and a shoulder at around $223 \mathrm{~nm}$, which is characteristic of the $\alpha$-helix conformation and in close agreement with the spectra obtained by other researchers. ${ }^{35,36}$ The ratio of $\theta_{208} / \theta_{223}$ can be used as a qualitative measure of rhINS association. ${ }^{34}$ This is due to the antiparallel $\beta$ structure which forms when two monomers dimerize. This results in an increase of $\theta_{223}$ without an 
A
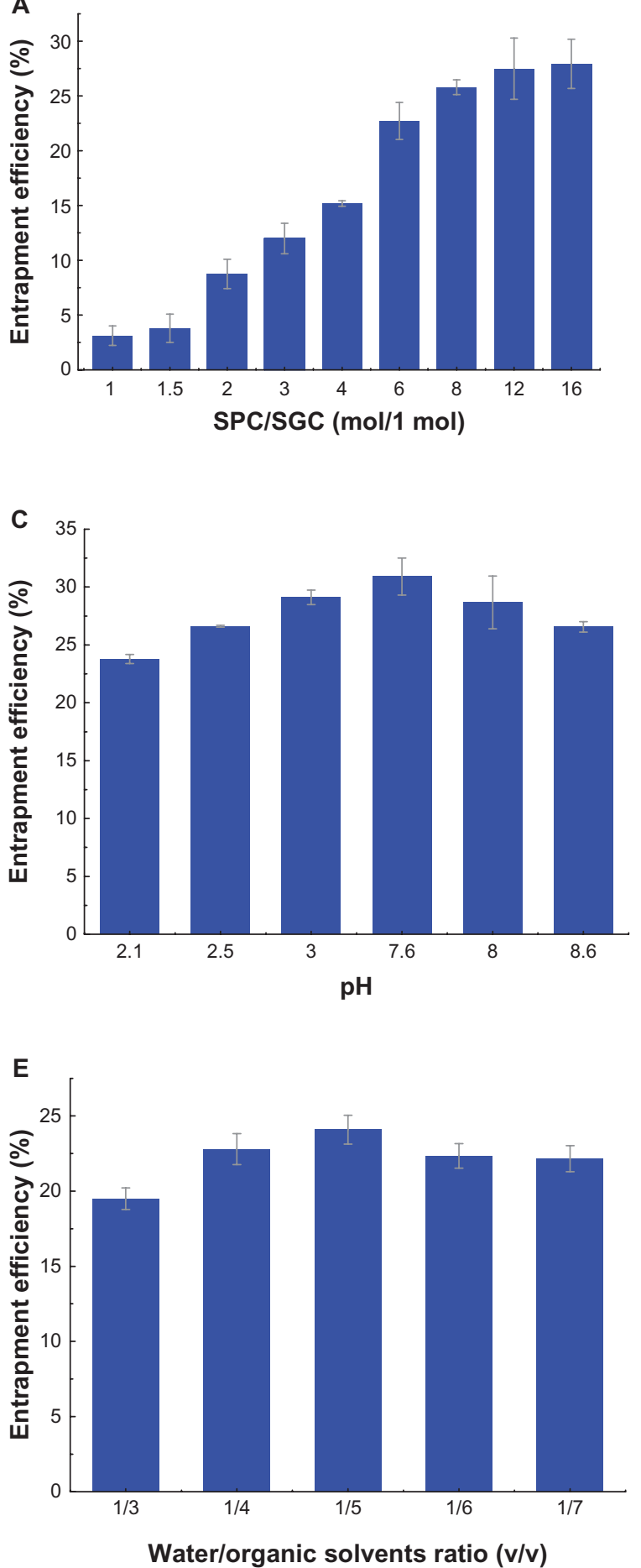

B

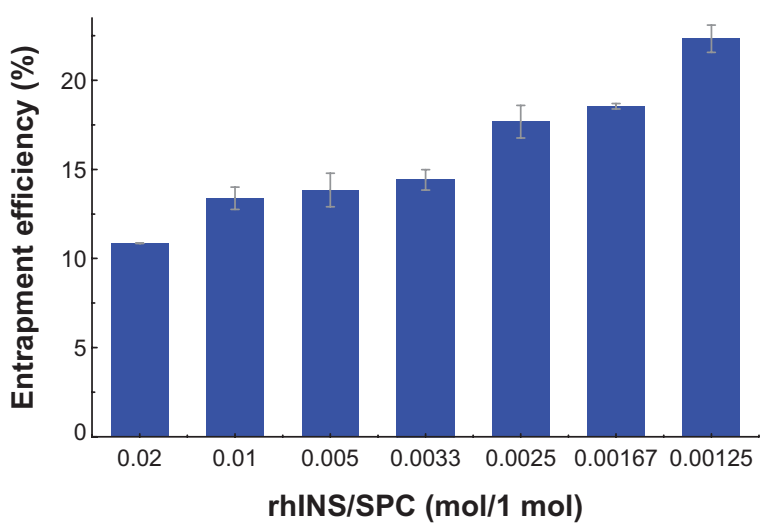

D

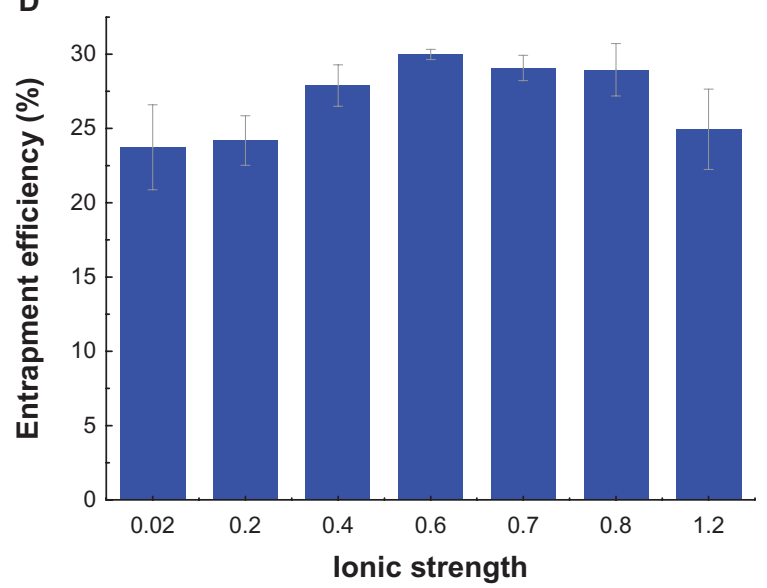

$\mathbf{F}$

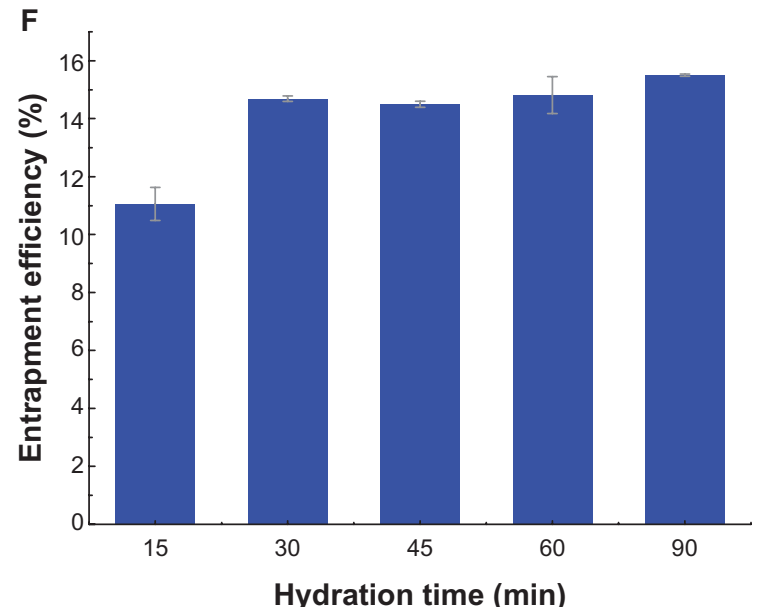

Figure 2 Effects of soybean phosphotidylcholine:sodium glycocholate ratio $(\mathbf{A})$, recombinant human insulin:soybean phosphotidylcholine ratio (B), water:ether volume ratio (C), $\mathrm{pH}$ of the inner water phase (D), ionic strength of the hydration buffer (E), and hydration time (F) on recombinant human insulin entrapment efficiency. Note: Data expressed as means \pm standard deviations $(n=3)$.

Abbreviations: SPC, soybean phosphotidylcholine; SGC, sodium glycocholate; rhINS, recombinant human insulin.

increase of $\theta_{208}$. The $\theta_{208} / \theta_{223}$ ratios of rhINS released from the six sodium glycocholate liposomes were within the range of 1.638-1.852, which was close to that of free rhINS (1.734, $P>0.05)$. Moreover, the helix ratio was $21.6 \%-24.5 \%$, which was in good agreement with that of natural rhINS $(23.3 \%, P>0.05)$. Such spectral features indicated that there was only a minor difference between the circular dichroism spectra of the sodium glycocholate liposome samples 


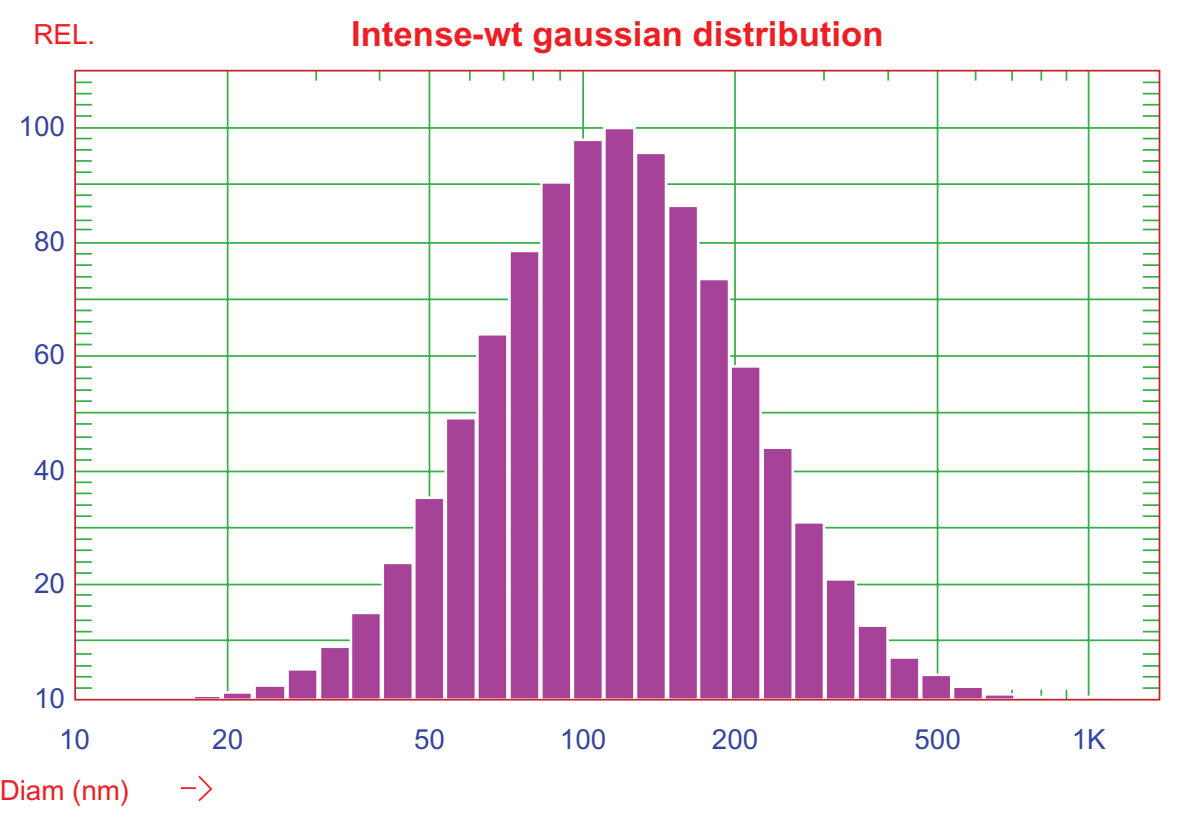

Figure 3 Particle size and distribution of recombinant human insulin-loaded sodium glycocholate liposomes prepared under optimal conditions.

prepared under different preparative conditions and that of the free rhINS solution.

The six sodium glycocholate liposome formulations prepared under extreme stress were disrupted by Triton X-100 to release rhINS, which was then administered to the mice subcutaneously at a dose of $0.5 \mathrm{IU} / \mathrm{kg}$. Blood glucose levels were then determined and are shown in Table 1. No significant difference $(P>0.05)$ was observed as compared with free rhINS solution, ie, the positive control, where a $53.1 \pm 12.6 \%$ reduction of the initial blood glucose level was obtained. However, a significant difference $(P<0.01)$ was observed for both the released rhINS and the positive control as compared with the negative control (blank sodium glycocholate liposomes). Therefore, it was concluded that the

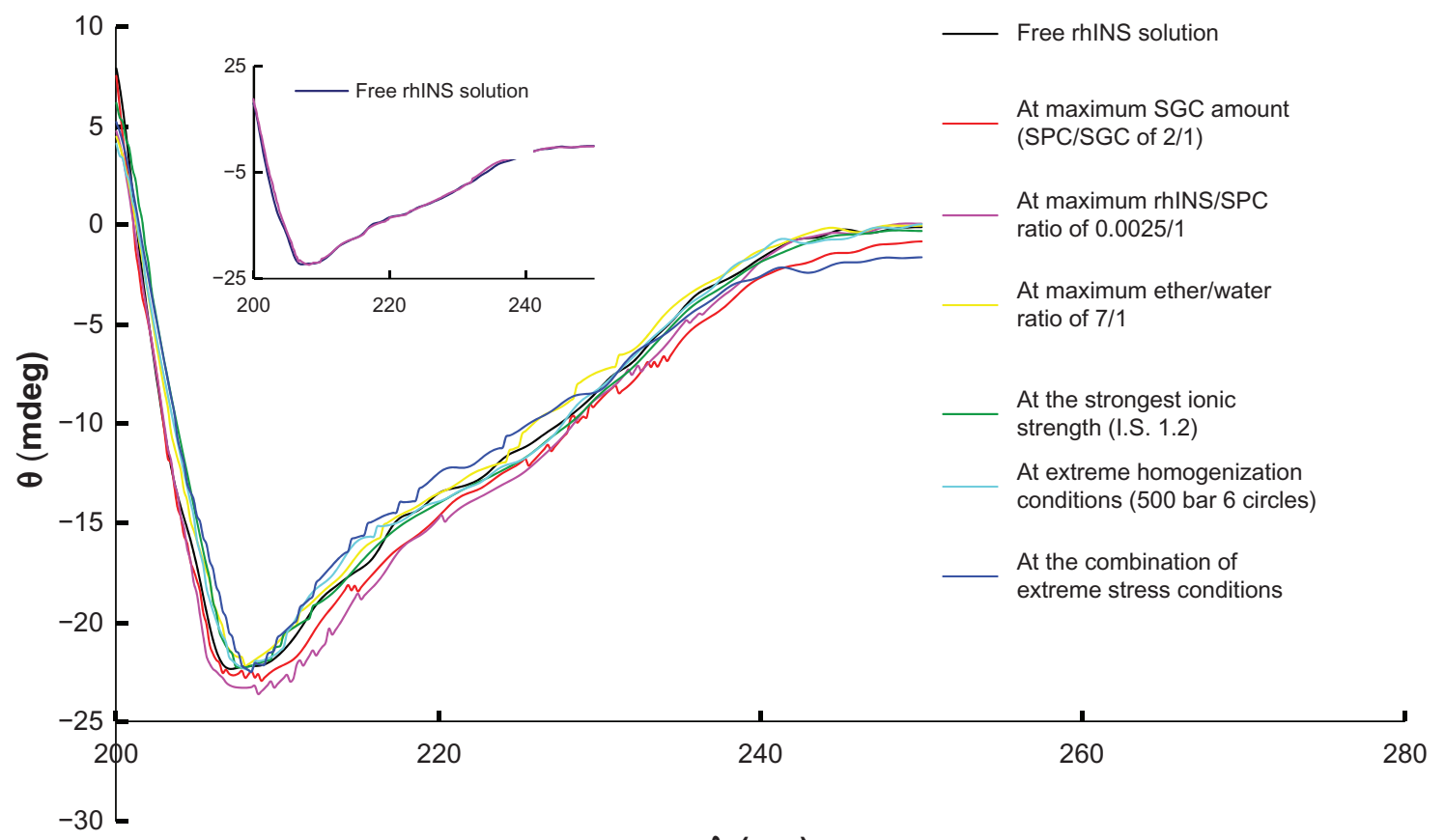

$\lambda(\mathrm{nm})$

Figure 4 Circular dichroism spectra of free insulin solution and insulin released from sodium glycocholate liposomes prepared under various extreme stress conditions at a recombinant human insulin concentration of $20 \mu \mathrm{g} / \mathrm{mL}$. The insert indicates circular dichroism spectrum of liposomal recombinant human insulin for the optimal formulation. Abbreviations: SPC, soybean phosphotidylcholine; SGC, sodium glycocholate; rhINS, recombinant human insulin; I.S., ionic strength. 
Table I Blood glucose level after subcutaneous administration of $0.5 \mathrm{lU} / \mathrm{kg}$ recombinant human insulin released from different sodium glycocholate liposomal formulations in mice. Data expressed as means \pm standard deviations $(n=6)$

\begin{tabular}{|c|c|c|c|}
\hline \multirow[t]{2}{*}{ SG liposome formulation } & \multicolumn{3}{|l|}{ Blood glucose level } \\
\hline & 0 hours $(\mathrm{mmol} / \mathrm{L})$ & I hour (mmol/L) & Reduction (\%) \\
\hline Free rhINS solution & $10.70 \pm 2.71$ & $4.96 \pm 1.44$ & $53.1 \pm 12.6$ \\
\hline Blank SGC liposomes & $9.93 \pm 1.59$ & $9.76 \pm 1.16$ & $1.2 \pm 6.3^{*}$ \\
\hline At maximum SGC amount (SPC:SGC ratio 2:I) & $10.98 \pm 2.74$ & $4.61 \pm 1.06$ & $57.7 \pm 3.9$ \\
\hline At maximum rhINS:SPC ratio of $0.0025: 1$ & $10.21 \pm 1.59$ & $4.35 \pm 0.50$ & $56.9 \pm 6.9$ \\
\hline At maximum ether:water ratio of $7: 1$ & $10.09 \pm 1.84$ & $5.16 \pm 1.39$ & $49.1 \pm 9.7$ \\
\hline At the strongest ionic strength (1.2) & $8.48 \pm 1.17$ & $3.83 \pm 1.28$ & $52.9 \pm 20.0$ \\
\hline At extreme homogenization conditions ( 500 bar, 6 cycles) & $7.94 \pm 1.35$ & $3.40 \pm 0.85$ & $57.3 \pm 6.4$ \\
\hline At combination of extreme stress conditions & $8.83 \pm 2.2$ & $3.78 \pm 0.69$ & $55.4 \pm 11.0$ \\
\hline
\end{tabular}

Note: $* P<0.05$, compared with free rhINS solution.

Abbreviations: rhINS, recombinant human insulin; SGC, sodium glycocholate; SPC, soybean phosphotidylcholine.

bioactivity of insulin was well preserved after drug loading, confirming the results of the conformational study.

The optimal formulation, which was prepared at less stress, showed good conformational stability (Figure 4, insert) and well preserved bioactivity, with a hypoglycemic percentage of $45.2 \%$ $\pm 6.5 \%$, which is similar to that of the free rhINS solution.

\section{Transmission electron microscopy}

Figure 5 shows the transmission electron micrographs of the blank and rhINS-loaded sodium glycocholate liposomes, as well as the conventional liposomes. An almost spheroidal morphology was observed for the conventional liposomes, whereas a less spheroidal and deformed morphology was observed for both blank and rhINS-loaded sodium glycocholate liposomes. This was possibly due to the deformability of the sodium glycocholate liposomes. The vesicular structure was discernable, and the inner lamella could be weakly observed for sodium glycocholate liposomes. No obvious changes in sodium glycocholate liposome morphology were observed as a result of drug loading.

\section{Leakage of insulin}

Figure 6 shows the variation in entrapment efficiency in $\mathrm{pH}$ 2.0, 5.6, and 6.8 buffers over 6 hours. There was no significant difference $(P>0.05)$ in entrapment efficiencies obtained at different time points and different $\mathrm{pH}$ values. The results
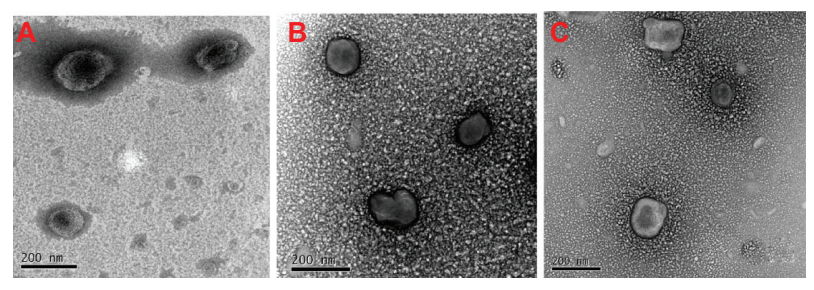

Figure 5 Transmission electron micrographs of conventional liposomes $(\mathbf{A})$, blank (B), and recombinant human insulin-loaded sodium glycocholate liposomes (C). indicate that leakage of rhINS from sodium glycocholate liposomes was slow within the observed time duration. Therefore, the effect of potential variations in entrapment efficiency can be ignored when interpreting the in vitro characterization results.

\section{Protection of insulin against digestion by pepsin, trypsin, and $\alpha$-chymotrypsin}

After treatment with pepsin, rhINS content in the conventional cholesterol liposomes was remarkably decreased from $100 \%$ to less than $5 \%$ of the initial value after 4 hours (Figure 7A). Liposomes containing bile salts all showed some resistance to pepsin because less degradation of rhINS was observed. Sodium glycocholate provided the best protection of liposomal rhINS, preserving $50 \%$ of rhINS at 4 hours. This may be attributable to the enzyme-inhibiting ability of sodium glycocholate. However, surprisingly, as the amount of sodium glycocholate increased further, less protection of

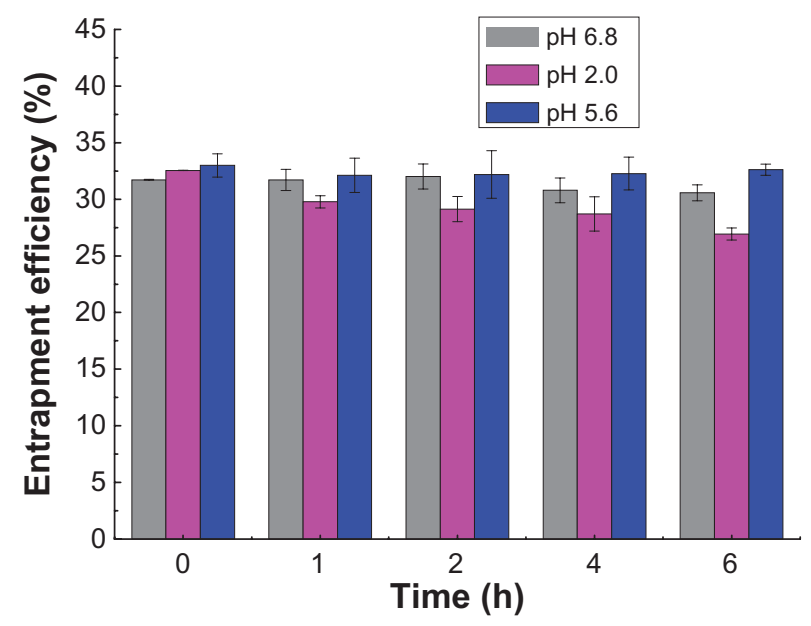

Figure $\mathbf{6}$ Leakage of recombinant human insulin from liposomes as measured by entrapment efficiency in buffers with different $\mathrm{pH}$ values.

Note: Data expressed as means \pm standard deviations $(n=3)$. 

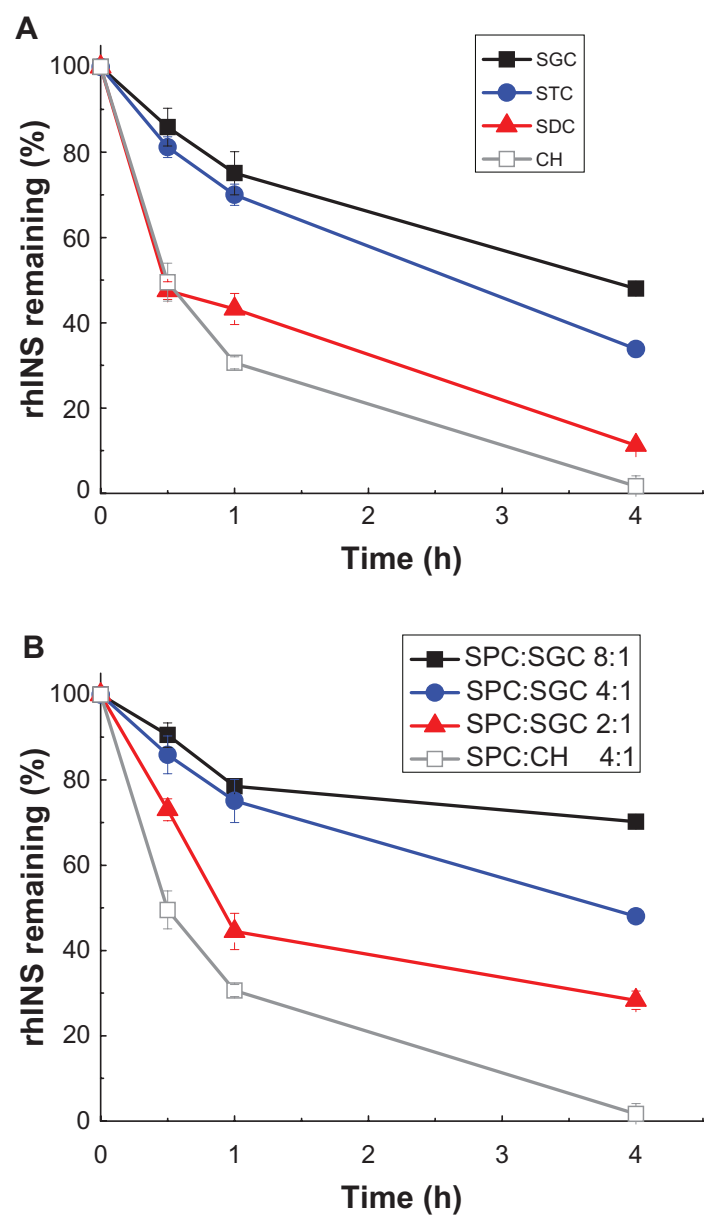

Figure 7 Protection of recombinant human insulin from pepsin degradation by liposomes with different types of bile salts, ie, sodium glycocholate, sodium taurocholate, sodium deoxycholate, (A) and different soybean phospholipid, ie, soybean phosphotidylcholine:sodium glycocholate ratios (B) for 4 hours at $37^{\circ} \mathrm{C}$. Note: Data expressed as means \pm standard deviations $(n=3)$.

Abbreviations: SPC, soybean phosphotidylcholine; SGC, sodium glycocholate; rhINS, recombinant human insulin; $\mathrm{CH}$, cholesterol.

liposomal rhINS was observed (Figure 7B). As the sodium glycocholate:soybean phosphotidylcholine ratio in the liposome formulation increased from 1:8 to $1: 2$, the amount of intact insulin decreased from $70 \%$ to $28 \%$ at 4 hours. This can possibly be explained by the increased flexibility of the lipid bilayers due to the presence of excessive amounts of sodium glycocholate, which may lead to the leakage of rhINS. The protective effect of bile salts on liposomal rhINS against trypsin and $\alpha$-chymotrypsin was different from that against pepsin. Conventional liposomes showed better resistance to trypsin than liposomes containing sodium taurocholate or sodium deoxycholate (Figure 8A). However, sodium glycocholate liposomes still exhibited the best protection against these two enzymes, preserving $67 \%$ and $81 \%$ of rhINS content at 4 hours for trypsin and $\alpha$-chymotrypsin, respectively
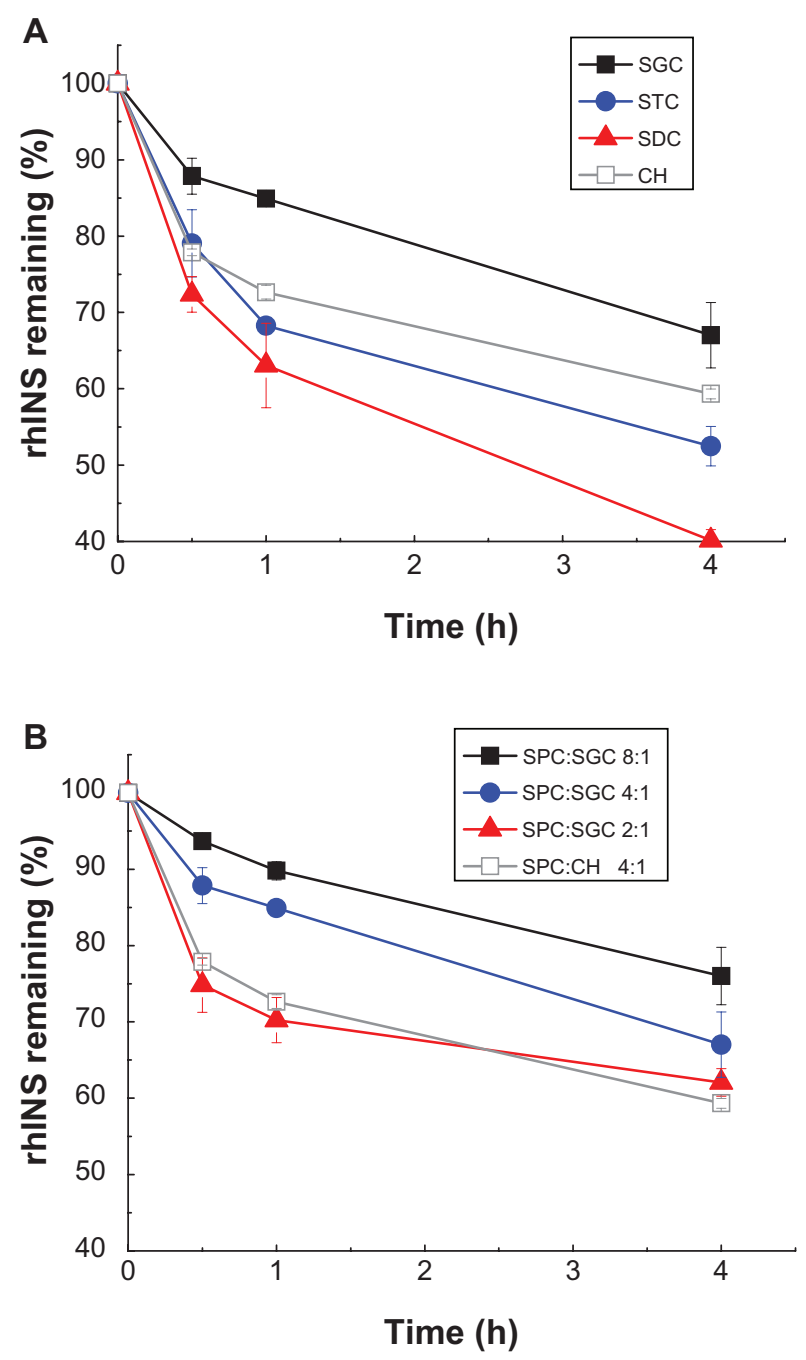

Figure 8 Protection of recombinant human insulin from trypsin degradation by liposomes with different type of bile salts, ie, sodium glycocholate, sodium taurocholate, sodium deoxycholate, (A) and different soybean phospholipid, ie, soybean phosphotidylcholine:sodium glycocholate ratios (B) for 4 hours at $37^{\circ} \mathrm{C}$.

Note: Data expressed as means \pm standard deviations $(n=3)$.

Abbreviations: SPC, soybean phosphotidylcholine; SGC, sodium glycocholate; rhINS, recombinant human insulin; $\mathrm{CH}$, cholesterol.

(Figures 8A and 9A). Again, an increase in the amount of sodium glycocholate in the liposome membrane leads to increased enzymatic degradation (Figures 8B and 9B).

\section{Discussion}

The application of liposomes containing bile salts in the field of oral immunization and oral delivery of poorly water-soluble drugs has triggered our interest in using this vehicle to deliver rhINS by the oral route. To achieve better protection against the detrimental gastrointestinal environment, we employed sodium glycocholate, a special bile salt that has been reported to have both enzyme-inhibiting and permeation-enhancing effects. ${ }^{28,30}$ Therefore, the purpose 

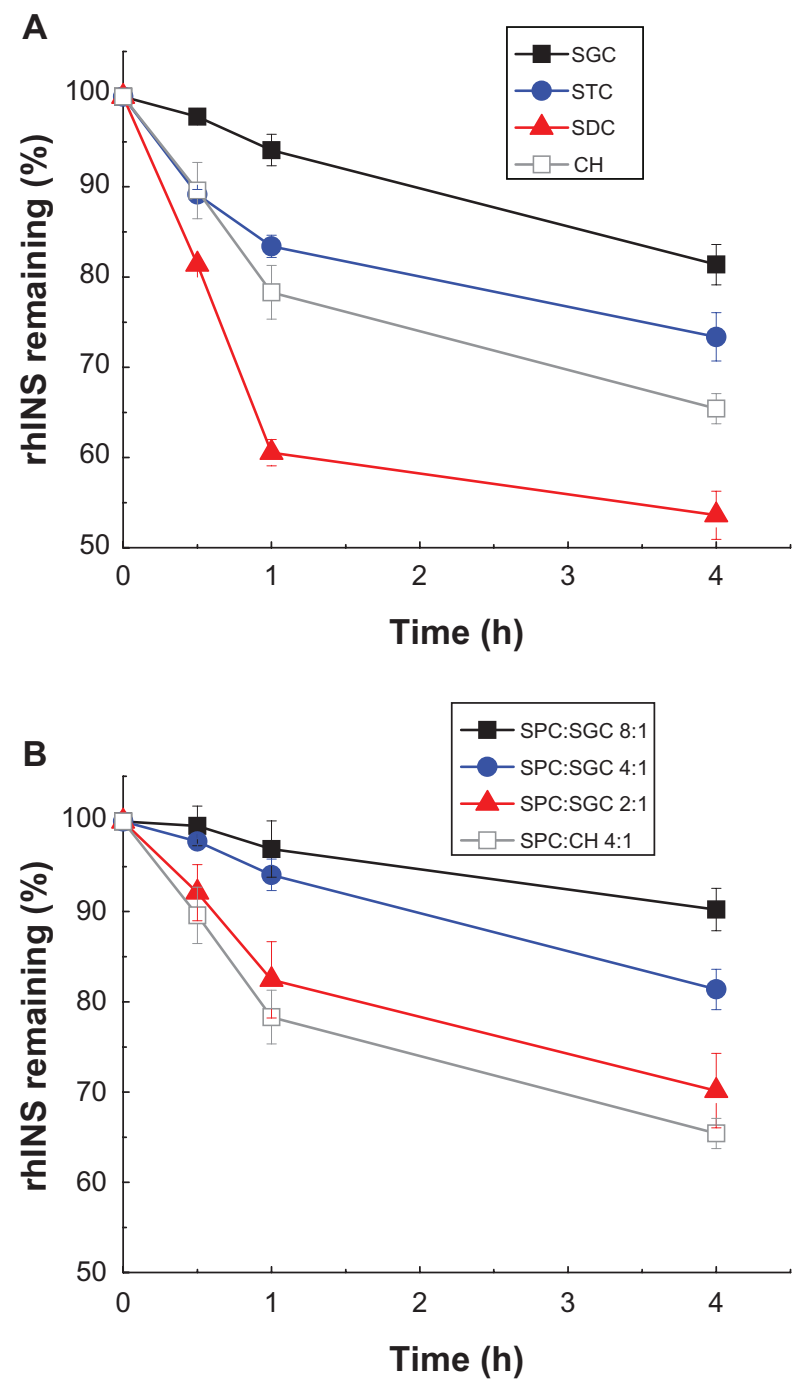

Figure 9 Protection of recombinant human insulin from $\alpha$-chymotrypsin degradation by liposomes with different type of bile salts, ie, sodium glycocholate, sodium taurocholate, sodium deoxycholate (A) and different soybean phospholipids, ie, soybean phosphotidylcholine:sodium glycocholate ratios (B) for 4 hours at $37^{\circ} \mathrm{C}$. Note: Data expressed as means \pm standard deviations $(n=3)$.

Abbreviations: SPC, soybean phosphotidylcholine; SGC, sodium glycocholate; rhINS, recombinant human insulin; $\mathrm{CH}$, cholesterol.

of this series of studies was to provide proof of concept of this new glycocholate-containing vehicle and to elucidate the underlying mechanisms. In this first report, we focus on issues of encapsulation of rhINS in sodium glycocholate liposomes and the protective effect of this vehicle against enzymatic degradation in simulated in vitro models. The hypoglycemic as well as mechanistic studies will be reported in upcoming publications.

The aim of the formulation study was to obtain liposomes with particle sizes as small as a few hundred nanometers, which, as previously reported, are very important in achieving good permeation across biomembranes. ${ }^{37}$ By increasing the homogenization pressure, the particle size can be easily reduced to as low as $100 \mathrm{~nm}$. However, reduction in particle size also leads to reduced entrapment efficiency of rhINS. Therefore, the homogenization pressure was finally settled at $300 \mathrm{bar}$, resulting in a particle size of about $150 \mathrm{~nm}$. Because the particle size can be adjusted easily using this method, we did not investigate the effect of other preparative variables on particle size.

The second goal of this formulation study was to achieve high rhINS loading. Through screening the critical process and formulation variables, an entrapment efficiency of rhINS in sodium glycocholate liposomes as high as $30 \%$ can be achieved for the optimal formulation. The objective of incorporating as much sodium glycocholate in the liposomes as possible was compromised by the fact that an excess of sodium glycocholate would lead to significant reduction in entrapment efficiency. This can be explained by the fluidizing effect of sodium glycocholate on the lipid bilayers, which probably results in leakage of rhINS. An increase in the rhINS:soybean phosphotidylcholine ratio was helpful to increase drug loading, but was at the expense of entrapment efficiency.

rhINS may be sensitive to interfacial stress during the ultrasonication or homogenization process. Therefore, it is very important to ensure preservation of rhINS stability in the final product. HPLC assay gives the chemical stability of rhINS content, whereas, conformational studies and bioactivity assays are usually utilized to ensure the integrity of rhINS. ${ }^{38,39}$ In this study, we investigated the conformational and bioactive stability of rhINS in liposomes prepared under extreme stress conditions. Both conformation, as measured by circular dichroism, and bioactivity was preserved. Therefore, we conclude that preparative stress does not cause destabilization of rhINS.

The clinical potential of liposomes containing bile salts would depend on their integrity in the gastrointestinal tract in order to achieve absorption-enhancing activity and protection of the rhINS. Thus, it is desirable not to release rhINS into the gastrointestinal tract. Therefore, we evaluated in vitro release for a duration of 6 hours by measuring the change in entrapment efficiency of sodium glycocholate liposomes. The $\mathrm{pH}$ of the release medium used in this section was 2, 5.6, and 6.8, which are the $\mathrm{pH}$ of the gastric environment, isoelectric point of rhINS, and intestinal environment, respectively. It is designed to evaluate the stability of the liposome in the gastrointestinal environment compared with that on the shelf; however, for protection from the enzyme degradation component, the $\mathrm{pH}$ values were settled at 1.2, 6.8, and 7.8, which were the most appropriate $\mathrm{pH}$ values for pepsin, trypsin, and $\alpha$-chymotrypsin. Stability of entrapment 
efficiency confirmed little leakage of rhINS. However, the integrity of sodium glycocholate liposomes needs to be studied in vivo.

The bile salts used could be either sodium glycocholate, sodium taurocholate, ${ }^{20}$ or sodium deoxycholate. ${ }^{40,41}$ These three bile salts were compared to highlight the effect of sodium glycocholate as an enzyme inhibitor. The in vitro protective effect against enzymatic degradation by pepsin, trypsin and $\alpha$-chymotrypsin, the three main proteases in the gastrointestinal tract, confirmed the advantage of glycocholate. Moreover, the safety of sodium glycocholate was better. As bile salts are naturally secreted by the gall bladder, the sodium glycocholate concentration in our formulation (6.77 $\mathrm{mM}$ ) was far lower than the toxic concentration of bile salts $(30 \mathrm{mM})$ for destroying the integrity/viability of Caco-2 monolayer cells. ${ }^{42}$

\section{Conclusion}

rhINS can be loaded into sodium glycocholate liposomes with high efficiency by a reversed-phase evaporation method followed by homogenization. The sodium glycocholate liposome particle size can be easily adjusted by tuning the homogenization pressure. The stability of rhINS was well preserved, even at extreme stress conditions, as confirmed by a conformational and bioactivity study. The formulation prepared under optimal conditions showed an entrapment efficiency of $30 \pm 2 \%$ and a particle size of $154 \pm 18 \mathrm{~nm}$. In vitro study of the protective effect of bile salts on liposomal rhINS against enzymatic degradation by pepsin, trypsin, and $\alpha$-chymotrypsin suggest superiority of sodium glycocholate. It is concluded that sodium glycocholate liposomes have potential for use in the oral delivery of protein and peptide drugs.

\section{Acknowledgment}

Novo Nordisk is acknowledged for providing recombinant human insulin and financial support for this study.

\section{Disclosure}

The authors report no conflicts of interest in this work.

\section{References}

1. Florence AT, Jani PU. Particulate delivery: The challenge of the oral route. Pharmaceutical Particulate Carriers. New York: Marcel Dekker; 1993.

2. Goldberg M, Gomez-Orellana I. Challenges for the oral delivery of macromolecules. Nat Rev Drug Discov. 2003;2(4):289-295.

3. Shah RB, Ahsan F, Khan MA. Oral delivery of proteins: Progress and prognostication. Crit Rev Ther Drug Carrier Syst. 2002;19(2): $135-169$.
4. Morishita M, Peppas NA. Is the oral route possible for peptide and protein drug delivery? Drug Discov Today. 2006;11(19-20):905-910.

5. Radwan MA, Aboul-Enein HY. The effect of absorption enhancers on the initial degradation kinetics of insulin by $\alpha$-chymotrypsin. Int $J$ Pharm. 2001;217(1-2):111-120.

6. Kamei N, Mariko M, Eda Y, Ida N, Nishio R, Takayama K. Usefulness of cell-penetrating peptides to improve intestinal insulin absorption. $J$ Control Release. 2008;132(1):21-25.

7. Sonaje K, Lin KJ, Wang JJ, et al. Self-assembled pH-sensitive nanoparticles: A platform for oral delivery of protein drugs. Adv Funct Mater. 2010;20(21):3695-3700.

8. Michaela KM, Andreas BS. Oral peptide drug delivery: Polymer-inhibitor conjugates protecting insulin from enzymatic degradation in vitro. Biomaterials. 2000;21(14):1499-1507.

9. Simon M, Behrens I, Dailey L, Wittmar M, Kissel T. Nanosized insulincomplexes based on biodegradable amine-modified graft polyesters poly[vinyl-3-(diethylamino)-propylcarbamate-co-(vinyl acetate)-co(vinyl alcohol)]-graft-poly(l-lactic acid): Protection against enzymatic degradation, interaction with Caco-2 cell monolayers, peptide transport and cytotoxicity. Eur J Pharm Biopharm. 2007;66(2):165-172.

10. Yin L, Ding J, He C, Cui L, Tang C, Yin C. Drug permeability and mucoadhesion properties of thiolated trimethyl chitosan nanoparticles in oral insulin delivery. Biomaterials. 2009;30(29):5691-5700.

11. Sonaje K, Chen YJ, Chen HL, et al. Enteric-coated capsules filled with freeze-dried chitosan/poly(gamma-glutamic acid) nanoparticles for oral insulin delivery. Biomaterials. 2010;31(12):3384-3394.

12. Lin YH, Sonaje K, Lin KM, et al. Multi-ion-crosslinked nanoparticles with $\mathrm{pH}$-responsive characteristics for oral delivery of protein drugs. J Control Release. 2008;132(2):141-149.

13. Mi FL, Wu YY, Lin YH, et al. Oral delivery of peptide drugs using nanoparticles self-assembled by poly(gamma-glutamic acid) and a chitosan derivative functionalized by trimethylation. Bioconjug Chem. 2008;19(6):1248-1255.

14. Hashimoto T, Nomoto M, Komatsu K, Haga M, Hayashi M. Improvement of intestinal absorption of peptides: Adsorption of B1-Phe monoglucosylated insulin to rat intestinal brush-border membrane vesicles. Eur J Pharm Biopharm. 2000;50(2):197-204.

15. Gao Y, He L, Katsumi H, Sakane T, Fujita T, Yamamoto A. Improvement of intestinal absorption of insulin and water-soluble macromolecular compounds by chitosan oligomers in rats. Int $J$ Pharm. 2008;359(1-2):70-78.

16. Cui FD, Tao AJ, Cun DM, Zhang LQ, Shi K. Preparation of insulin loaded PLGA-Hp55 nanoparticles for oral delivery. J Pharm Sci. 2007;96(2):421-427.

17. Thirawong N, Thongborisute J, Takeuchi H, Sriamornsak P. Improved intestinal absorption of calcitonin by mucoadhesive delivery of novel pectin-liposome nanocomplexes. J Control Release. 2008;125(3):236-245.

18. Song KH, Chung SJ, Shim CK. Enhanced intestinal absorption of salmon calcitonin ( $\mathrm{sCT}$ ) from proliposomes containing bile salts. J Control Release. 2005;106(3):298-308.

19. Zhang N, Ping QN, Huang GH, Xu WF. Investigation of lectin-modified insulin liposomes as carriers for oral administration. Int $J$ Pharm. 2005;294(1-2):247-259.

20. Degim Z, Unal N, Essiz D, Abbasoglu U. The effect of various liposome formulations on insulin penetration across Caco-2 cell monolayer. Life Sci. 2004;75(23):2819-2827.

21. Hanato J, Kuriyama K, Mizumoto T, et al. Liposomal formulations of glucagon-like peptide-1: Improved bioavailability and anti-diabetic effect. Int J Pharm. 2009;382(1-2):111-116.

22. Shukla A, Katare OP, Singh B, Vyas SP. M-cell targeted delivery of recombinant hepatitis $\mathrm{B}$ surface antigen using cholera toxin B subunit conjugated bilosomes. Int J Pharm. 2010;385(1-2):47-52.

23. Conacher M, Alexander J, Brewer JM, et al. Oral immunisation with peptide and protein antigens by formulation in lipid vesicles incorporating bile salts (bilosomes). Vaccine. 2001;19(20-22): 2965-2974. 
24. Singh P, Prabakaran D, Jain S, Mishra V, Jaganathan KS, Vyas SP. Cholera toxin B subunit conjugated bile salt stabilized vesicles (bilosomes) for oral immunization. Int J Pharm. 2004;278(2):379-390.

25. Shukla A, Khatri K, Gupta PN, Goyal AK, Mehta A, Vyas SP. Oral immunization against hepatitis B using bile salt stabilized vesicles (bilosomes). J Pharm Pharm Sci. 2008;11(1):59-66.

26. Senior K. Bilosomes: The answer to oral vaccine delivery? Drug Discov Today. 2001;6(20):1031-1032.

27. Schubert R, Jaroni H, Schoelmerich J, Schmidt KH. Studies on the mechanism of bile-salt-induced liposomal membrane damage. Digestion. 1983;28(3):181-190.

28. Song KH, Chung SJ, Shim CK, et al. Preparation and evaluation of proliposomes containing salmon calcitonin. $J$ Control Release. 2002;84(1-2):27-37.

29. Fetih G, Lindberg S, Itoh K, et al. Improvement of absorption enhancing effects of n-dodecyl-beta-D-maltopyranoside by its colon-specific delivery using chitosan capsules. Int J Pharm. 2005;293(1-2):127-135.

30. Shah RB, Palamakula A, Khan MA. Cytotoxicity evaluation of enzyme inhibitors and absorption enhancers in Caco-2 cells for oral delivery of salmon calcitonin. J Pharm Sci. 2004;93(4): 1070-1082.

31. Shao Z, Li Y, Krishnamoorthy R, Chermak T, Mitra AK. Differential effects of anionic, cationic, nonionic, and physiologic surfactants on the dissociation, alpha-chymotryptic degradation, and enteral absorption of insulin hexamers. Pharm Res. 1993;10(2):243-251.

32. Yomota C, Yoshii Y, Takahata T, Okada S. Separation of B-3 monodesamidoinsulin from human insulin by high-performance liquid chromatography under alkaline conditions. J Chromatogr A. 1996;721(1): 89-96.
33. Wei SJ, Smith NB. Improvements in the preparation of liposomes for electron microscopy and electronspectroscopic imaging (ESI). Microsc Res Tech. 1994;27(6):557-558.

34. Chen Y, Lu Y, Chen J, et al. Enhanced bioavailability of the poorly water-soluble drug fenofibrate by using liposomes containing a bile salt. Int J Pharm. 2009;376(1-2):153-160.

35. Pocker Y, Biswas SB. Conformational dynamics of insulin in solution: Circular dichroic studies. Biochemistry. 1980;19(22):5043-5049.

36. Tiyaboonchai W, Woiszwillo J, Sims RC, Middaugh CR. Insulin containing polyethylenimine-dextran sulfate nanoparticles. Int J Pharm. 2003;255(1-2):139-151.

37. Müller B, Kreuter J. Enhanced transport of nanoparticle associated drugs through natural and artificial membranes - a general phenomenon? Int J Pharm. 1999;178(1):23-32.

38. Pourhosseini PS, Saboury AA, Najafi F, Sarbolouki MN. Interaction of insulin with a triblock copolymer of PEG-(fumaric-sebacic acids)PEG: Thermodynamic and spectroscopic studies. Biochim Biophys Acta. 2007;1774(10):1274-1280.

39. Sadhale Y, Shah JC. Stabilization of insulin against agitation-induced aggregation by the GMO cubic phase gel. Int J Pharm. 1999;191(1): 51-64.

40. Kim SK, Lee DY, Lee E, et al. Absorption study of deoxycholic aidheparin conjugate as a new form of oral anti-coagulant. $J$ Control Release. 2007;120(1-2):4-10.

41. Lee S, Kim K, Kumar TS, et al. Synthesis and biological properties of insulin-deoxycholic acid chemical conjugates. Bioconjug Chem. 2005; 16(3):615-620.

42. Johansson F, Hjertberg E, Eirefelt S, Tronde A, Hultkyvist BU. Mechanisms for absorption enhancement of inhaled insulin by sodium taurocholate. Eur J Pharm Sci. 2002;17(1-2):63-71.
International Journal of Nanomedicine

\section{Publish your work in this journal}

The International Journal of Nanomedicine is an international, peerreviewed journal focusing on the application of nanotechnology in diagnostics, therapeutics, and drug delivery systems throughout the biomedical field. This journal is indexed on PubMed Central, MedLine, CAS, SciSearch ${ }^{\circledR}$, Current Contents ${ }^{\circledR} /$ Clinical Medicine,

\section{Dovepress}

Journal Citation Reports/Science Edition, EMBase, Scopus and the Elsevier Bibliographic databases. The manuscript management system is completely online and includes a very quick and fair peer-review system, which is all easy to use. Visit http://www.dovepress.com/ testimonials.php to read real quotes from published authors. 